INTERNATIONAL JOURNAL OF

ARTIFICIAL INTELLIGENCE AND EMERGING

TECHNOLOGY

\title{
Virtual Reality Technology and its Role in Advertising Field
}

\author{
Ahmed Abdel Azem Mahmoud a, *
}

a Instructor in photography, cinema and television Department, Faculty of Applied Arts - October 6 University

\begin{abstract}
The technological development has made many scientific revolutions in various fields and produced many of the means and methods of modern devices that have become part of our daily lives and for the field of motion picture and the emergence of technological digital revolution in this field has become the change quickly producing a digital image with different characteristics on both sides of technical and digital technology did not stop at this point but have evolved to make the viewer part of the digital system and so the appearance of the various interactive systems that did the role of the viewer moving from the recipient only to the participant through the introduction of new devices called Virtual Reality systems. The virtual reality is one of modern technology that used in many different fields, there are many different types of virtual reality systems but they all share the same characteristics such as the ability to allow the person to view three dimensional images.
\end{abstract}

Keywords

Head-Mounted Display

Artificial World

Simulation

Virtual Environment

Technology

\section{Introduction}

The technological development has made many scientific revolutions in various fields and produced many of the means and methods of modern devices that have become part of our daily lives and for the field of motion picture and the emergence of technological digital revolution in this field has become the change quickly producing a digital image with different characteristics on both sides of technical and digital technology did not stop at this point but have evolved to make the viewer part of the digital system and so the appearance of the various interactive systems that did the role of the viewer moving from the recipient only to the participant through the introduction of newdevices 
called Virtual Reality systems . The virtual reality is one of modern technology that used in many different fields, there are many different types of virtual reality systems but they all share the same characteristics such as the ability to allow the person to view threedimensional images.

\section{Research problem}

There are many problems associated with the research topic, namely:

1. The emergence of some virtual reality images Far from realitydue to the lack of choice of appropriate technological means.

2. The lack of connection between the real lighting methods and the lighting methods used in the software and computers to obtain a picture of the reality of the convergence of the real picture in terms of lighting.

3. The spread of virtual reality technology significantly in games, programs and advertisements. Therefore, the methods of production of the virtual reality image and the technology of real images and lighting should be noted and linked. 
INTERNATIONAL JOURNAL OF

ARTIFICIAL INTELLIGENCE AND EMERGING

TECHNOLOGY

\section{Research goal}

The research aims at many points including:

1. clarify the concepts and terminology, systems and devices for virtual reality.

2. Identify the state of the digital modern technology methods and devices used in virtual reality systems for digital image in advertisingfield. 3. Shed light on the scientific importance of virtual reality in different uses, beside the entertainment .

\section{Research Methodology:}

the research Followed by the descriptive analytical method based on the definition and description and analysis of the virtual reality and its devices used, and the latest technology in this field.

\section{Research Results and recommendations}

1- The use of virtual reality technology has become imperative in many fields, so it must be carefully studied to obtain the highestpossible benefit from it.

2- The use of virtual reality technology in advertising has openednew Prospects in this field.

3- A collective action plan should be developed among all participants in the virtual reality technology industry to obtain thehighest possible quality.

4- The area of virtual reality technology should be increased in many areas, especially in the fields of education, medicine and engineering.

5- Virtual reality technology should be included in the Academic studies that dealing with digital image production. 


\section{What is Virtual Reality?}

Virtual reality can be defined as an upcoming technology that makes users feel in a Virtual Environment (VE) by using computer hardware and software It was originally conceived as a digitally created space which humans could access by donning special computer equipments. It enables people to deal with information more easily. VR provides a different way to see and experience information ${ }^{(1)}$.

VR has been defined as a realistic and immersive simulation of a threedimensional environment, created using interactive software and hardware, and experienced or controlled by movement of the body or as an immersive, interactive experience generated by a computer.

A person using virtual reality equipment is typically able to "look around" the artificial world, move about in it and interact with features or items that are depicted on a screen or in goggles. Most 2016-era virtual realities are displayed either on a computer monitor, a projector screen, or with a virtual reality headset also called head-mounted display or HMD ( Fig 1). HMDs typically take the form of head-mounted goggles with a screen in front of the eyes ${ }^{(\top)}$.
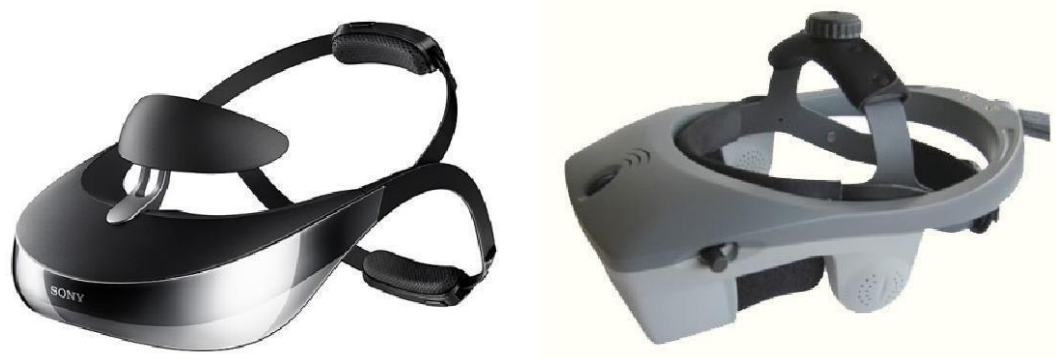

(Fig. 1) head-mounted display

To enter in a VE, a user dons special gloves, earphones, and goggles, all of which send their output to the computer systems. The virtual environments are intended to replace the real world environment with the digital one and the human senses are immersed in the VE. Immersion is an experience of losing oneself in the VE and shutting out all cues from 
the physical world. A Virtual Environment can be created on different extents depending on the computer based platform ranging from a cell phone screen to a desktop monitor or a fully Immersive Virtual Environment (IVE) $)^{(1)}$.

The definition of virtual reality comes, naturally, from the definitions for both 'virtual' and 'reality'. The definition of 'virtual' is near and reality is what we experience as human beings. So the term 'virtual reality' basically means 'near-reality'. This could, of course, mean anything but it usually refers to a specific type of reality emulation.

We know the world through our senses and perception systems. In school we all learned that we have five senses: taste, touch, smell, sight and hearing. These are however only our most obvious sense organs. The truth is that humans have many more senses than this, such as a sense of balance for example. These other sensory inputs, plus some special processing of sensory information by our brains ensures that we have a rich flow of information from the environment to our minds.

Everything that we know about our reality comes by way of our senses. In other words, our entire experience of reality is simply a combination of sensory information and our brains sense-making mechanisms for that information. It stands to reason then, that if you can present your senses with made-up information, your perception of reality would also change in response to it. You would be presented with a version of reality that isn't really there, but from your perspective it would be perceived as real. Something we would refer to as a virtual reality ${ }^{(1)}$.

\section{The history of virtual reality}

Before the 1950s the exact origins of virtual reality are disputed, partly 
because of how difficult it has been to formulate a definition for the concept of an alternative existence. Elements of virtual reality have surfaced as early as the 1860s with French playwright Antonin Artaud who used avantgarde work to blur illusion and reality to be one and the same.The first references to the more modern concept of virtual reality came from science fiction. Stanley G. Weinbaum's 1935 short story "Pygmalion's Spectacles" describes a goggle-based virtual reality system with holographic recording of fictional experiences, including smell and touch.

Morton Heilig wrote in the 1950s of an "Experience Theatre" that could encompass all the senses in an effective manner, thus drawing the viewer into the onscreen activity. He built a prototype of his vision dubbed the Sensorama in 1962 (Fig. 2) along with five short films to be displayed in it while engaging multiple senses (sight, sound, smell, and touch). Predating digital computing, the Sensorama was a mechanical device. The patent application described the device as "a telescopic television apparatus for individual useThe spectator is given a complete sensation of reality, i.e. moving three dimensional images which may be in colour, with $100 \%$ peripheral vision, binaural sound, scents and air breezes".

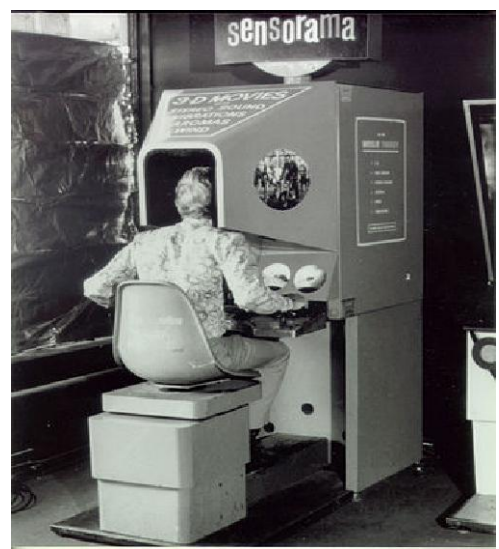

(Fig. 2) The Sensorama was released in the 1950s. 
By the 1980s the term "virtual reality" was popularized by Jaron Lanier, one of the modern pioneers of the field. Lanier had founded the company VPL Research in 1985. VPL Research has developed several VR devices like the Data Glove, the Eye Phone, and the Audio Sphere. VPL licensed the Data Glove technology to Mattel, which used it to make an accessory known as the Power Glove.

In 2010, Palmer Luckey designed the first prototype of the Oculus Rift. This prototype, built on a shell of another virtual reality headset, was only capable of rotational tracking. However, it boasted a 90-degree field of vision that was previously unseen in the consumer market at the time. This initial design would later serve as a basis from which the later designs came (fig. 3).

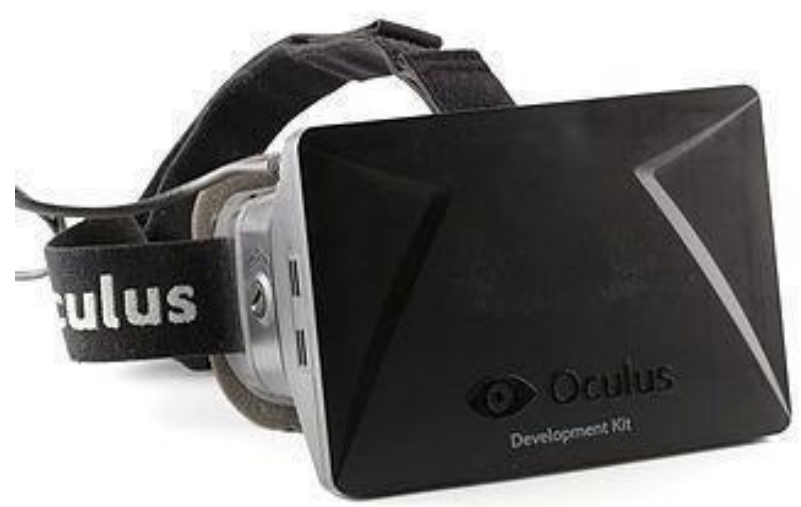

(fig. 3) developer version of Oculus Rift from Oculus VR.

In 2013, Valve discovered and freely shared the breakthrough of lowpersistence displays which make lag-free and smear-free display of VR content possible. This was adopted by Oculus and was used in all their future headsets.

In early 2014, Valve showed off their SteamSight prototype, the precursor to both consumer headsets released in 2016. It shared major features with the consumer headsets including separate $1 \mathrm{~K}$ displays per eye, low persistence, positional tracking over a large area, and fresnel lenses.

On March 25, 2014, Facebook purchased Oculus VR for \$2 billion. This purchase occurred before any of the devices ordered through Oculus' 
2012 Kickstarter had shipped

In that same month, Sony announced Project Morpheus (its code name for PlayStation VR).

By 2016 there were at least 230 companies developing VR-related products. Facebook had 400 employees focused on VR development, and Google, Apple, Amazon, Microsoft, Sony and Samsung all had dedicated $A R$ and VR groups. Dynamic binaural audio was common to most headsets released that year. Visually, displays were still of a low- enough resolution and frame-rate that images were still identifiable as virtual. On April 5, 2016, the first units of the HTC VIVE SteamVR headset began shipping. This marked the first major commercial release of sensor based tracking, allowing for free movement of users within a defined space. In early 2017, a patent filed by Sony showed they were developing a similar location tracking technology to the VIVE for PlayStation VR, with the potential for the development of a wireless headset ${ }^{(1)}$.

\section{Lighting for virtual reality}

What is light? Visible light is really a very small portion in a range of electromagnetic radiation. This radiation travels in waves of different wavelengths. The difference in wavelengths (the "tops" of each wave) is what makes the difference between blue, red, gamma rays, $x$-rays, radio waves etc (Fig. 4).
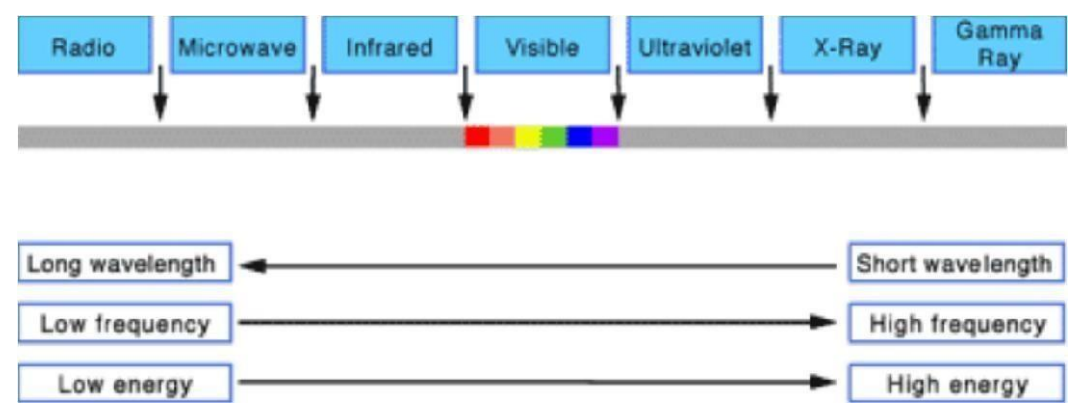

(Fig. 4) Electromagnetic spectrum( ${ }^{(1)}$.

"White light" is a combination of all colours in the visible spectrum. When we perceive an object as red for example, what really happens is that 
white light falls on a red surface, and all the wavelengths except those that give red light are absorbed by the material. Only the red portion of the spectrum is reflected back.

Developing photorealistic virtual environments (VE), for example 3D animations or games or VE used in rehabilitation or therapy, we need to focus not only on the principle of ecological validity: modelling objects and environments, using good texture, real like objects and environments, which fit the users' needs, but we have to take care of using correct lighting, too. What kind of light is correct in a scene? What is the quality of light? The answer would be defined by: softness, intensity, colour, and attenuation. What is photorealistic lighting? Most people do not realize that every light source has distinct colour, based on its colour temperature. We do not actually see this colour because our eyes automatically adapt to changes in colour temperature. Our eyes automatically correct the colour of the light so that white objects look white under a large variety of yellowish white to bluish white lights. This has to be included in the rendering program. On the other hand, if we would like to emphasize the situation in which the observer sees the scene, as e.g. whether we render a street lighting scene in fog or at dawn, some further considerations are necessary, and the present techniques are not always optimal to do such renderings. Lighting a scene in $3 D$ is much like lighting a scene for photography, film or theatre. As an element of design, light must be considered a basic influencing factor at the beginning of the creative process and not something to be added later.Colour and lighting is the most critical question and also one of the most complicated things in developing $3 \mathrm{D}$ environments. We have to understand relevant aspects of optics,

physics, computer science, human perception and arts too. In the real world for an artist it does not matter if his/her hands and clothes are dirty with the paints or a sculptor sees the final statue in a rough stone with his/her mind's eye. A 3D animator or software engineer has to know everything about the renderer that he/she uses.

\section{Lighting in MAYA software}

Lighting a Maya scene is much like lighting a scene for photography, film, or theatre. As an element of design, light must be considered a basic influence at the beginning of the creative process and not something to be added later. This is especially true in computer graphics where 
lighting is based on mathematical algorithms, and creating real world lighting effects can require a solid understanding of the software application

\section{Lighting concepts}

The basic premise of good lighting is appropriate design. Light gives objects or characters "meaning" in their surroundings. It also provides an appropriate and intentional atmosphere that will be logically interpreted by the viewer.

The design potential of light is inherent in its physical characteristics. By controlling its intensity, colour, and direction, light becomes a key factor in creating a scene. Lighter and darker areas help to compose the frame and guide the eye towards certain objects and actions.

\section{Choosing light types}

Once we have determined the direction and distribution of lights on a scene, we will also need to consider the type of light source.Professional 3D software(s) provide a selection of different light types that all have attributes which can be edited and animated to simulate real world lighting. These lights can produce a range of qualities, form soft and diffuse to harsh and intense because they each have different characteristics. While it is likely that our combination of lights and techniques will vary with each production, the design principles of combining hard and soft edged light, different angles, intensities, and shadows remain the same.

This article shows the following types of virtual reality lighting facilities:

- Directional lights

- Point lights

- Ambient lights 
- Spot lights

- Area lights

- Volume lights (1)

\section{Methods for bringing Virtual Reality to life}

In this part we will talk about achieving realism in 3D images through lighting, but first a quick disclaimer we will be focusing on "realism," which is not to be confused with "reality," or "the real world," or whatever phrase you use to describe the magical dance of energy that engulfs us all. We will leave it to the philosophers to tackle what is "real," and instead focus on what makes 3D images look real to the viewer. There are 5 types of lighting for 3D products

\section{1- Ambient Light}

Also known as available light, ambient light is the light already available in the environment. This is similar to natural light. Imagine pulling light curtains closed over a large window. The room becomes evenly lit with no harsh shadows or highlights, which makes everything in the room easy to see because your eyes don't need to adjust as you look around. Game engines can easily fake this kind of light on low-end hardware such as mobile devices. It's an easy way to brighten a virtual scene without taxing the rendering pipeline. But be careful. The simplicity of virtual ambient light has a major drawback: It doesn't cast shadows. This can make the scene look strange, and if you turn ambient light up too much things start to look unnatural and cartoony( fig. 5 ). 


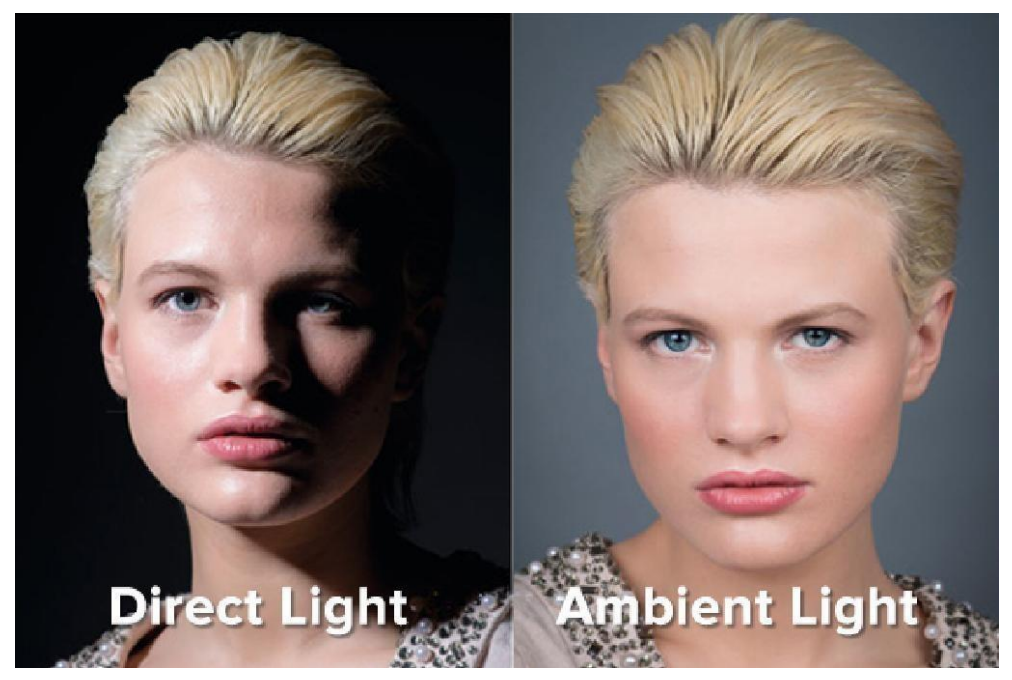

(fig. 5) ambient light and direct light

\section{2- Shadows}

The example with ambient light demonstrates how important shadows are to making a realistic scene. When an object has a shadow, it means it's solid and has presence in the scene. A floating box without a shadow looks very out of place. It looks like a hologram, floating in the air. You might have trouble telling how far away or how big the box is. By adding a shadow it gives the box presence. You can immediately see the box is quite large and in the distance. Shadows are a good addition to virtual scenes. The type of shadow also gives hints about lighting and environment. Hard shadows, with hard edges, indicate a point light. Dark shadows indicate a bright light source with little ambient light. ( fig. 6 ) 


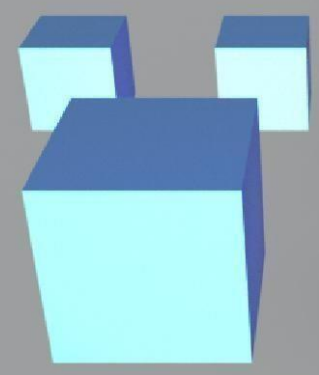

No Shadows

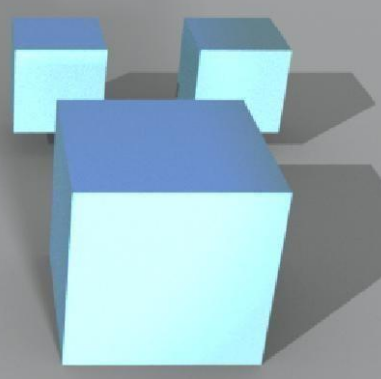

Shadows

( fig. 6 ) shadows

\section{3- Ambient Occlusion}

An ambient occlusion is a special type of shadow. The name describes what is going on: The ambient light gets occluded, or blocked, by nearby objects. You can often see this in action in corners. Look into corners and you will notice they are darker than the surrounding area. This is actually quite taxing for a computer to do. Desktop and laptop computers can do it in real time, but only high-end mobile devices can handle it. One workaround is to use baked ambient occlusion on objects. Unfortunately, in that case, the object casting the shadow cannot move relative to the surface it'son. ( fig. 7 ) 

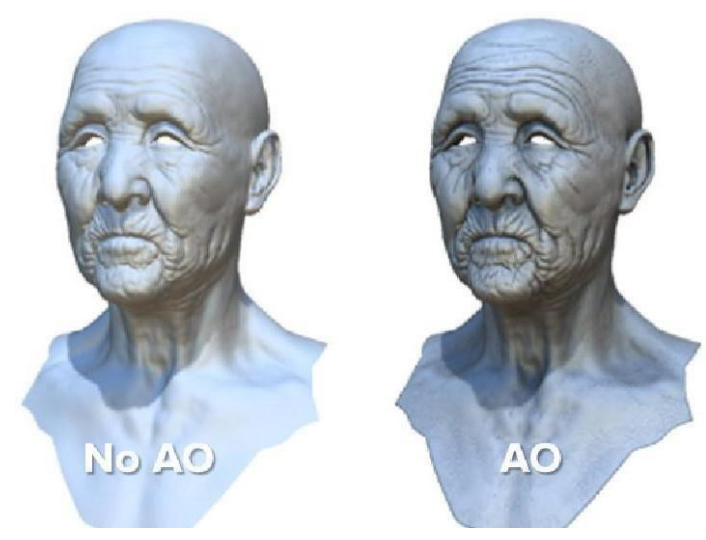

( fig.7 ) Ambient Occlusion

\section{4- Specularity}

The way light interacts with objects is crucial for believing those objects are real. Does the object reflect light? Does light pass through it? Does it give off its own light? Is it some combination of the above? Light interacts differently with plastic as compared to something like skin. You can distinguish wood and steel by how light reflects and scatters off of it. The most common property is specularity. ( fig. 8 )

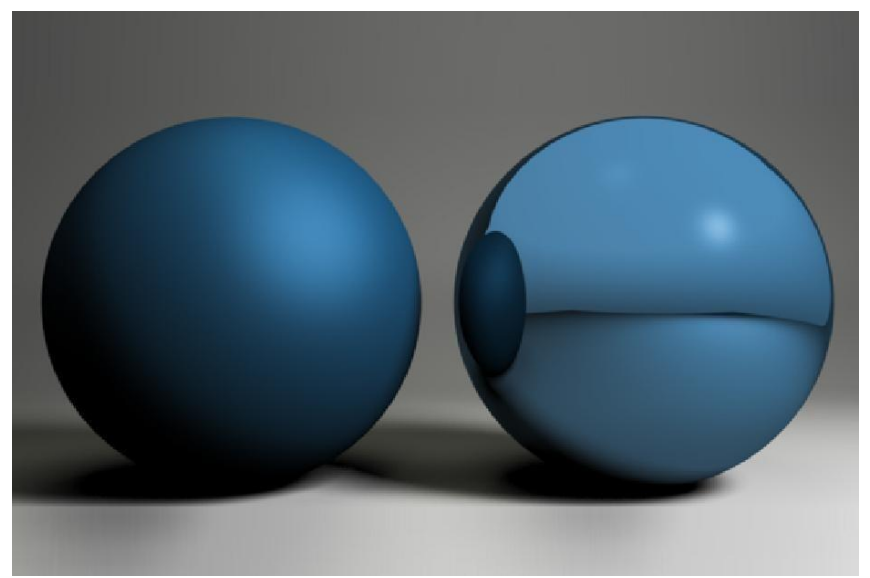


(fig. 8) Specularity5-

Physically Based Rendering

All the described properties and more are taken into account in physically based rendering (PBR). Legacy rendering methods used simple properties such as diffuse color and specular amount to approximate realism. PBRs, on the other hand, uses actual properties taken from real world objects. This makes achieving realism less time consuming, because many materials already have known values for the properties in PBR. And if they are unknown, they are easy to measure and plug in the values (fig. 9) (1).

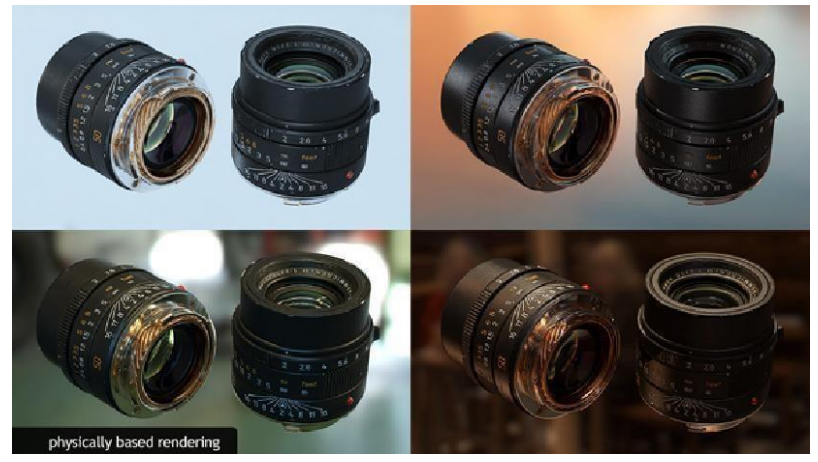

(fig. 9) Physically Based Rendering

\section{Virtual reality in Marketing}

Virtual reality presents a unique opportunity for advertisers to reach a completely immersed audience. Companies such as Paramount Pictures, Coca-Cola, McDonald's and Disney have applied VR into marketing campaigns. Non-profit organizations such as Amnesty International, UNICEF, and World Wide Fund for Nature (WWF) have used virtual reality to bring potential supporters closer to their work, effectively bringing distant social, political and environmental issues and projects to members of the public in immersive ways not possible with traditional media. Panoramic 360 views of conflict in Syria and face to 
face encounters with CGI tigers in Nepal have been used in experiential activations and shared online for educational and fundraising purposes $\left({ }^{(1)}\right.$.

Lowe's, IKEA, Wayfair and other retailers have developed systems that allow their products to be seen in virtual reality, to give consumers a better idea of how the product will fit into their home, or to allow the consumer to get a better look at the product from home. Consumers looking at digital photos of the products can "turn" the product around virtually, and see it from the side or the back.

Several companies develop software or services that allow architectural design firms and real estate clients to tour virtual models of proposed building designs. During the design process, architects can use VR to experience the designs they are working on before they are built.

Seeing a design in VR can give architect a correct sense of scale and proportion. VR models can replace physical miniatures to demonstrate a design to clients or the public. Developers and owners can create VR model of built spaces that allow potential buyers or tenants to tour a space in VR, even if real-life circumstances make a physical tour unfeasible.

In July 2015, OnePlus became the first company to launch a product using virtual reality. This was for their second flagship device the OnePlus 2, first viewable using OnePlus Cardboard, based on Google Cardboard platform. It was accessed through an app released on Google Play Store, then on YouTube (").

\section{Lighting for Virtual Reality Filmmaking}

Lighting shots in virtual reality filmmaking requires a whole new way of creative thinking. The basic premise of good lighting is appropriate design, but how do you decide what is appropriate when the audience can decide where to set the point of view, the 'virtual frame' of the camera at all times? In this post, we're exploring lighting for $360^{\circ}$ Virtual Reality films.

Traditionally, DOP's and lighting craftsmen will approach a scene from the basis of 3-point lighting. Experienced operators tend to move on, 
but this fundamental lighting style forms the backbone of many a cinematographic education. The problem is, 3-point lighting requires lights, often big lights, which sit in the middle of the room behind the camera.

These lights tend to come in a variety of flavours including area lights, spot, directional, volume, ambient - each has their own purpose for crafting the look of a scene. But a lot of these tools simply aren't going to work in VR without accepting a break of the immersion factor.

Obviously pointing a spot light at a subject such as an actor is going to draw attention to that object, especially if that is the intention, and using a fill of some variety will bring the level of lighting in a place to an acceptable level for the camera sensor, but these lights can't simply be hidden out of shot.

As VR filmmakers we don't have the freedom of using lights hidden behind us, so arguably we are going to have to decide if we are going to hide lights in shot, or just accept the user is going to see them if they look into the corners or up to the ceiling. I'm ok with this as a creative choice, but it will have the effect of taking the subject out of the scene.

Even the most creative VR filmmaker is going to have difficulty convincing a user the 10k HMI peeking over the top of a treeline isn't meant to be there. In instances such as this, perhaps a panoramic film makes more sense to give the crew room to hide kit in zeniths and nadirs.

Hiding lights is probably the way forward, but it doesn't have to be as simple as placing a sofa in front of the kino. Getting creative with the practical side of lighting will help massively. Build kino-flos into sets as if they belong there in the production design is going to become more common. Perhaps having extensive amounts of tungsten lamps dotted 
INTERNATIONAL JOURNAL OF

ARTIFICIAL INTELLIGENCE AND EMERGING

TECHNOLOGY

around a room will be a common lighting approach. We're simply going to have to come up with new more naturally physical lights which aren't black, boxy and cumbersome.

In the below example from Millers Crossing, there are a number of practicals used to motivate the lighting, but in VR, those practicals will become the only way to paint the scene with light, making them the focus and most important light sources in the room (fig. 10 ). 
INTERNATIONAL JOURNAL OF

ARTIFICIAL INTELLIGENCE AND EMERGING

TECHNOLOGY

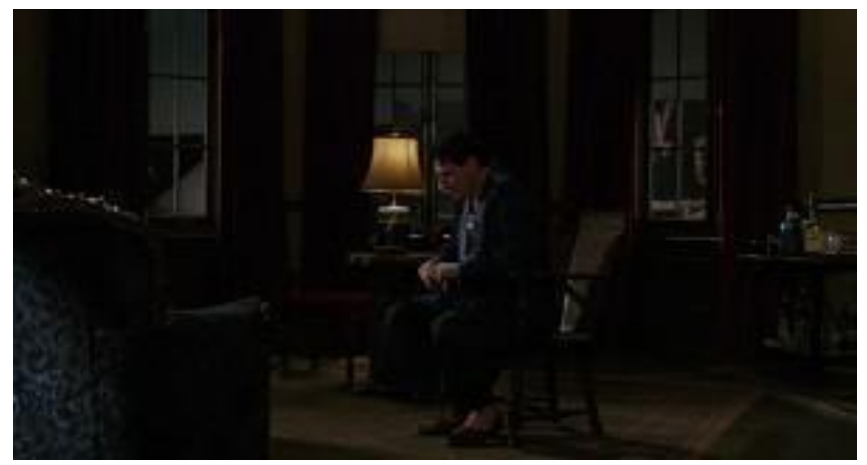

(fig. 10) example from Millers Crossing

At the moment, a lot of $360^{\circ} \mathrm{VR}$ films shoot outside, simply due to the bright ball in the sky offering up all the flat and even light that is needed. As VR filmmaking develops, we're expecting smaller, more powerful lights to be more effectives as they can be hidden more easily or even designed to be modularly built into practical designs such as lamps. As with many aspects of virtual reality filmmaking, it's pretty much a whole new language to learn ${ }^{(1)}$.

\section{Virtual Advertising}

Virtual Advertising is the use of digital technology to insert virtual advertising images into a live or pre-recorded television show, often in Sports events. This technique is often used to allow broadcasters to replace real advertising panels (existing on the playfield) with virtual images on the screen when broadcasting the same event in other regions which are not concerned with the local advertising; a Spanish football game will be broadcast in Mexico with Mexican advertising images. The viewer has the impression that the advertising image he/she sees on screen is the one in the reality.

The technology used in virtual insertions often uses automated 
processes such as: automatic detection of playfield limits, automatic detection of cuts, recognition of playfield surface, etc.

An operator is usually dedicated to the visual control of the effect but new systems allow using the instant replay operator.

On YouTube, we can see Virtual Advertising, early bet on 360-degree video. This means viewers can see the video from every angle just by swiping or moving the phone or tablet around-no headset required. Uploads of 360-degree videos continue to grow and have doubled over the past three months. 2 Brands are forging the way, using 360-degree video to film big events or get creative with ads. BMW used this technology for an ad featuring a 360-degree car race. The "School of Rock" musical created a 360-degree music video. AT\&T simulated a car crash to drive home its phone safety message ${ }^{(1)}$.

\section{Virtual Advertising applications}

Virtual advertisements can be effectively integrated into live television in real-time. For example, Fox Sports Net places a virtual advertisement on the glass behind the goaltender that can only be seen on television. In baseball Major League Baseball places virtual advertisements on a backboard behind the batter which can be targeted differently in local markets or countries. During the World Series MLB International broadcasts the World Series with different advertisements on a per market basis, showing a different ad in the US, Canadian, Latin American and Japanese markets. In Formula One, virtual ads are placed on the grass or as virtual billboards.

\section{Virtual Product Placement}

The technology that enabled video tracking for virtual advertising has been used for the past decade to create virtual product placements in television shows hours, days, or years after they have been produced. This presents several benefits including the possibility of preserving an advertisement-free master copy of the show in order to generate 
revenue as product placements are added in the future. Advertisements can be targeted to regional markets and updated over time to ensure maximum efficiency of advertising money.There are some systems used by virtual reality such as:

- Broadcast Virtual Virtual Advertising software and services

- ORAD AdVision Virtual advertising media platform

- The BrandMagic Virtual Advertising systems and services

- Epsio from EVS Broadcast Equipment

- Supponor DBRLive Virtual Advertising for sporting event (1)

\section{4-D Light Field Displays}

The first experience with a prototype of the Oculus Rift, they have been getting more and more excited about high quality consumer virtual reality hardware. The first production version of the Rift is almost here, and when it arrives (probably in early 2016), you might even be able to justify its rumored $\$ 1,500$ cost.

Good as the Rift is, it's taken this long for Oculus to get the hardware ready because fooling your eyes and brain to the extent that the Rift (or any other piece of VR hardware) does is a very tricky thing to pull off. The vast majority has an entire lifetime of experience of looking at the world in 3$D$, and we notice immediately when things aren't quite right. This can lead to headaches, nausea, and a general desire never to try VR ever again. A big part of what makes VR so difficult is that it's continually trying to convince your eyes that they should be focusing on a scene in the distance, when really, they're looking at a screen just a few inches away.

The current generation of VR displays use a few different techniques to artificially generate images that appear to have depth despite being 
displayed on flat screens. But there's one that they're missing out on one that could make VR displays much more comfortable to use. The same sort of 4-D light field technology that allows the Lytro camera to work its magic could solve this problem for VR as well.

Conventional VR displays like the Oculus Rift artifically create images that seem to have depth using visual depth cues including:

Binocular Disparity (Stereopsis) Your left and right eyes each see a slightly different scene. When your brain processes these two scenes, the disparities between the two scenes give the illusion of depth.

Motion Parallax - As you move your head from side to side, things that are closer to your eyes appear to move faster laterally than things that are farther from your eyes. Displays take advantage of that to make your brain think some objects in the scene are farther away than they actually are.

Binocular Occlusions Objects that are in the foreground of a scene, and are therefore in front of other objects, are nearer. This results in a simple relative ranking of distance. But if the amount of occlusion is different for each eye, the brain registers it as depth.

Vergence When your eyes look at something, the closer that thing is to you, the more your eyes have to rotate inwards (converge) to keep it in the center of your field of view. If something is farther away, your eyes have to rotate outwards (diverge) until at an infinite distance, the center of gaze for your eyes are parallel. The amount of rotation gives your brain the data it needs (two angles and the base of a triangle) to calculate the distance to the thing. 
To take advantage all of these depth cues, most VR displays show a separate image to each eye. If those images are properly synced up with each other and with the motion of your head, it generates a tolerably convincing illusion of depth.

However, your brain can still tell that something's not quite right, because vergence is coupled with another depth cue: focus. When something close to you is in focus, your eyes are converged a bit and as you focus on things that are farther away, your eyes diverge. Most head-mounted virtual reality displays (HMDs) don't present focus cues at all. The entire image is always in focus, because it's on a flat screen that stays at the same distance from your eyes. But, your eyes are converging and diverging depending on the part of a changing stereoscopic image your attention is drawn to Your brain doesn't like this at all, and this "vergenceaccommodation conflict" can result in "visual discomfort and fatigue, eyestrain, diplopic vision, headaches, nausea, compromised image quality, and it may even lead to pathologies in the developing visual system of children." So say the Stanford University researchers who will be presenting a paper at SIGGRAPH 2015. They, of course, have a way of fixing this problem: $\underline{4-} \underline{D}$ light field displays.

To understand what a 4-D light field display is and how it works, it helps to start from the other side of the process, with a 4-D light field camera. You can buy one of these; they're funny looking and made by a company called Lytro. We've got a fantastic explainer about how Lytro's light field camera works, but essentially, it's like putting a whole bunch of tiny little cameras together to capture light from multiple perspectives of a scene simultaneously. By recording the direction that each light ray comes from, you can do a bunch of fancy math to reconstruct how that scene will look at any point of focus. The 4-D, incidentally, refers to the 
dimensional information contained in each pixel: $\mathrm{x}$ location, $\mathrm{y}$ location, and $\theta$ and $\varphi$ for the angle of the incoming light ray.

So now you've got a camera that can capture images viewable from any perspective. Cramming those images into a display would be pretty awesome, if for no other reason than because it would solve the family of VR-induced problems that includes vergence- accommodation, visual discomfort and fatigue, eyestrain, diplopic vision, headaches, nausea, compromised image quality, and pathologies in the developing visual system of children.

And this is what the Stanford researchers have managed to put together: a high resolution light field display with refresh rates that makeit practical for $\mathrm{VR}^{(1)}$.

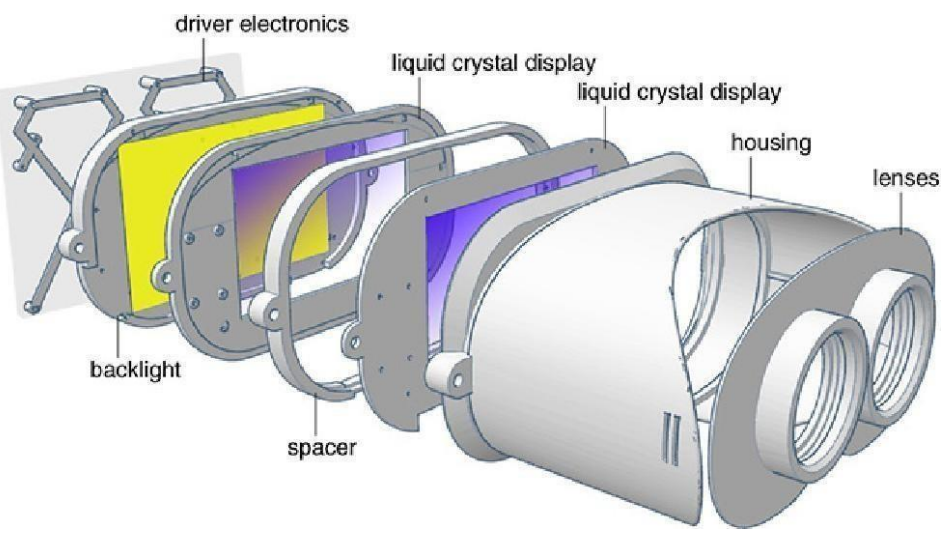

4-D Light Field Displays

\section{References}

- Choi,Dong Hwa," Emerging Tools and Applications of VirtualReality in Education", information science reference, 2016.

- Vincent G. Duffy, "Handbook of Digital Human Modeling",CRC press,2016.

- Steve Aukstakalnis, "Practical Augmented Reality", PearsonEducation, 


\section{INTERNATIONAL JOURNAL OF \\ ARTIFICIAL INTELLIGENCE AND EMERGING \\ TECHNOLOGY}

Limited, 2016.

- Peeter Verlegh،Hilde Voorveld‘Martin Eisend,"Advances

inAdvertising Research ", Springer Gabler,2016.

- https://en.wikipedia.org/wiki/Virtual_reality.

- https://www.engineersgarage.com/articles/virtual-reality-environment

- https://www.vrs.org.uk/virtual-reality/what-is-virtual-reality.html.

- $\quad$ http://science.howstuffworks.com/light3.htm.

- https://www.researchgate.net/.../228400406 Lighting in Virtual Reality.

- $\quad$ www.marxentlabs.com/shine-creating-realistic-3d-imagery-lighting.

- https://en.wikipedia.org/wiki/Virtual_reality.

- $\quad$ speedvr.co.uk/360-vr.../lighting-for-virtual-reality-filmmaking.

- $\quad$ spectrum.ieee.org/.../4d-light-field-displays-are-exactly-what-

virtual-reality

- https://en.wikipedia.org/wiki/Virtual_advertising.

https://www.thinkwithgoogle.com/.../virtual-reality-

advertising.html 